\title{
Tratamiento de efluentes de la industria minera con dolomita
}

\author{
${ }^{(1)}$ Alfonso A. Romero \\ ${ }^{(2)}$ Silvana L. Flores \\ ${ }^{(3)}$ Walter Arévalo
}

\begin{abstract}
RESUMEN
El artículo presenta una metodología de tratamiento de las aguas ácidas obteniendo la remediación de los efluentes de la industria minera, basado en el empleo de la dolomita tratada que permite reducir las concentraciones de los iones de metales pesados disueltos en los efluentes minero-metalúrgicos a valores altos de reducción de los metales pesados, los cuales son indicadores de una mejora en la calidad de agua de los desechos industriales provenientes, principalmente, de procesos metalúrgicos de plantas concentradoras.

Esta técnica de tratamiento resuelve el problema de generación de efluentes metalúrgicos con el empleo de la dolomita, mineral no metálico de carbonato doble de calcio y magnesio $\left(\mathrm{CaCO}_{3}\right.$. $\mathrm{MgCO}_{3}$ ), que se comporta como un efectivo reactivo de remediación, debido a las propiedades fisicoquímicas de la neutralización de la acidez de aguas ácidas, asi como la adsorción de iones metálicos disueltos en las aguas ácidas.

Asimismo, la aplicación de este tratamiento de remediación propone establecer una metodología de trabajo que desarrolle una tecnología de limpieza a nivel de laboratorio, con perspectivas a desarrollar la misma tecnología a nivel de planta piloto, con el posterior desarrollo de una planta de tratamiento de remediación a nivel industrial.
\end{abstract}

Palabras clave: Remediación, dolomita, efluentes de la industria minera, aguas ácidas.

EFLUENT TREATMENT WITH DOLOMITE MINING

\section{ABSTRACT}

The article shows a methodology for acid water treatment, through the remediation of effluents from the mining industry, based on the use of treated dolomite, which reduces the concentrations of dissolved heavy metal ions in metallurgical and mining effluent to reducing high levels of heavy metals, which are indicators of improved water quality of industrial wastes, mainly from metallurgical processes concentrator plants.

This treatment technique solves the problem of effluent generation with the use of dolomite, which is a nonmetallic mineral double calcium carbonate and magnesium $\left(\mathrm{CaCO}_{3} \cdot \mathrm{MgCO}_{3}\right)$, which acts as an effective reagent for remediation, because its physicochemical properties of the acid neutralizing acid water and adsorption of dissolved metal ions in acidic waters.

Moreover, the implementation of this remediation treatment, proposes to establish a working methodology to develop a cleaning technology at the laboratory, with prospects to develop clean technology to pilot plant level, with subsequent development of a treatment plant remediation at the industrial level.

Key words: Remediation, dolomite, mining industry effluents, acid water.

\section{INTRODUCCIÓN}

En el Perú, no existen antecedentes de tratamientos de remediación y purificación de efluentes metalúrgicos de flotación con el empleo de la dolomita natural y dolomita comercial, que busque reducir la concentración de iones de metales pesados, principales generadores de aguas ácidas.

El tratamiento de remediación con dolomita es un método alternativo de precipitación y purificación de estos efluentes, empleando la dolomita tratada, con la finalidad de reducir la concentración de metales pesados, tales como el cobre presente en los efluentes metalúrgicos, que son efluentes potenciales generadores de acidez que contaminan los cuerpos de agua.

\section{FUNDAMENTO TEÓRICO}

Por lo general, los métodos de tratamiento metalúrgicos se clasifican en métodos activos.

\subsection{Métodos activos}

Precisan de una operación continuada, como sucede en una planta química de tratamiento de aguas ácidas. El tratamiento de aguas de mina utilizando métodos químicos mediante la adición de sustancias alcalinas, tiene un coste elevado, sobre todo, cuando se trata de grandes volúmenes. Además requiere un control y el mantenimiento de las instalaciones de aireación y mezclado, así como de un almacenamiento adecuado de los lodos con carga metálica ${ }^{[5]}$.

Se puede afirmar que la mayoría de sistemas de tratamiento activo para aguas ácidas de mina se basan en la precipitación de hidróxidos, que se realiza en un proceso de tres pasos:

- Paso 1: Oxidación (para convertir $\mathrm{Fe}^{+2}$ en $\mathrm{Fe}^{+3}$ ).

- Paso 2: Dosis con álcalis, especialmente $\mathrm{Ca}(\mathrm{OH})_{2}$ pero también con $\mathrm{Na}(\mathrm{OH}) 2, \mathrm{NaHCO}_{3}$ y otras sustancias.

- Paso 3: Sedimentación.

Docentes de la Facultad de Ingeniería Geológica, Minera, Metalúrgica y Geográfica de la Universidad Nacional Mayor de San Marcos, Lima-Perú.

E-mail: aromerob@unmsm.edu.pe / Email: sflores.engineer.translater@gmail.com

Email: warevalog@unmsm.edu.pe 
Sin embargo, cabe resaltar que la información referente al tratamiento de purificación de efluentes metalúrgicos de flotación mediante el uso de dolomita, que involucre la reducción de la concentración de iones generadores de acidez, principalmente ión cobre, es escasa; debido a que casi no existen estudios ni informes técnicos referidos al tema [3].

Es en ese sentido que se busca lograr que las concentraciones de los principales iones metálicos disueltos estén por debajo de los valores de los Límites Máximos Permisibles establecidos por la Ley 27759 , Ley General de Aguas, Decreto Ley N. ${ }^{\circ} 17752$, para clase III, puesto que se desea evitar la generación de acidez de las aguas, en el área donde se desarrollan actividades minero-metalúrgicas, puesto que contaminan el suelo y los cuerpos de agua.

El objetivo principal del tratamiento de remediación con dolomita para la purificación de efluentes metalúrgicos, es lograr que se le considere como un método de tratamiento de las aguas ácidas generadas por efluentes metalúrgicos, y que permita: la supresión de la acidez, la precipitación de los metales pesados y la eliminación de sustancias contaminantes como sólidos en suspensión, arseniatos, antimoniatos y otros.

\subsubsection{Tratamiento de remediación de efluentes de la industria minera con dolomita}

Es una solución al problema de generación de aguas ácidas de los efluentes de la industria minera que provienen de los procesos metalúrgicos de flotación.

Este tratamiento alternativo se constituye en un eficaz método de remediación de efluentes metalúrgicos a un bajo costo debido a la abundancia de la dolomita.

La remediación se logra mediante las reacciones químicas espontáneas y simultáneas de neutralización, precipitación y adsorción de los iones metálicos disueltos de cobre que se producen durante la agitación de la mezcla [4].

La neutralización se produce por las propiedades fisicoquímicas de adsorción que posee la dolomita, un mineral no metálico de carbonato doble de calcio y magnesio $\left(\mathrm{CaCO}_{3} \cdot \mathrm{MgCO}_{3}\right)$ que conduce a la reducción de las concentraciones del ión metálico de cobre disuelto presente en los efluentes provenientes de procesos metalúrgicos de flotación hasta los valores cercanos a los límites máximos permisibles, los cuales son indicadores de una mejora en la ca- lidad de agua de los ríos que se ve afectada por el vertimiento de aguas de desechos industriales provenientes principalmente de procesos metalúrgicos de plantas concentradoras [2].

La dolomita es un mineral bastante común en las rocas sedimentarias continentales y marinas, se puede encontrar en capas de varios cientos de metros, y es uno de los minerales más difundidos en las rocas sedimentarias carbonatadas; se forma por la acción del agua rica en magnesio, sobre depósitos calcáreos, en donde se produce una progresiva substitución del calcio por el magnesio; a este proceso se le denomina dolomitización, y es un caso concreto de los procesos de sustitución en general o metasomatismo (sustitución de una sustancia por otra), también se forma por actividad hidrotermal [2].

$$
2 \mathrm{CaCO}_{3}+\mathrm{Mg}^{+2} \rightarrow \mathrm{CaMg}\left(\mathrm{CO}_{3}\right)_{2}+\mathrm{Ca}^{2+}
$$

El yacimiento de la dolomita natural se encuentra comprendido entre la cordillera central y oriental de la parte central del Perú.

En el área de estudio, conforman unidades geomorfológicas con colinas subandinas disectadas por sistemas de fallamientos inversos que generaron "Ventanas geológicas", las cuales ponen en contacto al grupo Pucará (Triásico-Jurásico) con formaciones más recientes del Cretáceo Superior y Mioceno.

\section{METODOLOGÍA DE INVESTIGACIÓN}

El Diseño Experimental del Proyecto de Investigación involucra la realización de actividades de gabinete y actividades de laboratorio.

\subsection{Actividades de gabinete}

Abarcan actividades de recolección de la información relacionada con el tratamiento de reducción de la concentración de iones generadores de acidez, principalmente ión cobre, que se encuentra presente en las aguas ácidas producidas por efluentes metalúrgicos.

Asimismo, se realizarán actividades del procesamiento de datos del trabajo y el análisis de los resultados. Estas actividades se realizan de manera previa y durante el trabajo experimental.

\subsection{Experimentos realizados}

El diseño experimental del proyecto de investigación involucra la realización de pruebas experi- 
mentales a nivel de escala de laboratorio, con la finalidad de lograr tanto la generación de efluentes metalúrgicos provenientes de procesos de flotación como la remediación de los mismos con la finalidad de reducir la concentración inicial de los principales iones generadores de acidez.

La realización de pruebas experimentales del tratamiento de remediación, basada en el empleo de la dolomita, hace énfasis en el estudio de las curvas de cinética de adsorción con dolomita de los efluentes metalúrgicos de flotación, el cual demuestra que la remediación con dolomita reduce la concentración de iones generadores de acidez que se dan a través de la etapa de adsorción de estos iones en la dolomita.

Es en ese sentido, que el estudio tiene como propósito determinar la efectividad del nuevo tratamiento.

El mineral de dolomita empleado en las pruebas experimentales del tratamiento de remediación son: dolomita natural y dolomita comercial.

Para la realización de las pruebas experimentales del tratamiento de remediación de efluentes metalúrgicos de flotación, se utilizó:

- Dolomita natural:

Malla $(-325)=100 \%$.

- Dolomita comercial:

Retenido en malla No $325(\%)$ : 0.1+-0.5\%.

- Efluente metalúrgico de flotación de la planta concentradora de la UNI. Se realizaron pruebas a partir de un volumen total de 20 litros.

Variables independientes del tratamiento de remediación:

- Concentración inicial de metales pesados disueltos en el efluente metalúrgico de flotación: $0.34 \mathrm{~g} / \mathrm{Lt}$.

- Tiempo de agitación de la mezcla (efluente metalúrgico con dolomita): 0-30 minutos

\section{Variables dependientes de remediación}

- Concentración final de metales pesados de cobre disueltos en el efluente metalúrgico: varía, llegando a valores cercanos a los límites máximos permisibles
3.2.1. Caracterización química y mineralógica de muestras de dolomita natural y dolomita comercial

\subsubsection{Análisis químico de la dolomita natural}

Tabla 1. Análisis químico típico.

\begin{tabular}{|l|c|}
\hline \multicolumn{1}{|c|}{ Componente } & $\begin{array}{c}\text { Composición química } \\
(\%)\end{array}$ \\
\hline $\mathrm{SiO} 2$ & 0.15 \\
\hline $\mathrm{CaO}$ & 30.43 \\
\hline $\mathrm{MgO}$ & 21.18 \\
\hline $\mathrm{Al} 2 \mathrm{O} 3$ & 0.06 \\
\hline $\mathrm{TiO} 2$ & $<0.01$ \\
\hline $\mathrm{MnO}$ & 0.05 \\
\hline $\mathrm{P} 2 \mathrm{O} 5$ & 0.107 \\
\hline $\mathrm{SrO}$ & $<0.01$ \\
\hline $\mathrm{BaO}$ & $<0.01$ \\
\hline $\mathrm{Pérdida} \mathrm{por} \mathrm{calcinación}$ & 46.7 \\
\hline Fe2O3 & 1.1 \\
\hline
\end{tabular}

Fuente: Flores S. (2009). Tratamiento de Remediación de Efluentes Metalúrgicos con énfasis en el abatimiento de cobre con dolomita. Tesis para obtener Titulo Profesional de Ingeniero Metalúrgico de la Universidad Nacional Mayor de San Marcos. ${ }^{[6]}$

\subsubsection{Análisis químico de la dolomita comercial}

Tabla N.² 2. Análisis químico típico

\begin{tabular}{|l|c|}
\hline \multicolumn{1}{|c|}{ Componente } & $\begin{array}{c}\text { Composición química } \\
(\%)\end{array}$ \\
\hline $\mathrm{SiO} 2$ & 0.15 \\
\hline $\mathrm{CaO}$ & 30.43 \\
\hline $\mathrm{MgO}$ & 21.18 \\
\hline $\mathrm{Al} 2 \mathrm{O} 3$ & 0.06 \\
\hline $\mathrm{TiO} 2$ & $<0.01$ \\
\hline $\mathrm{MnO}$ & 0.05 \\
\hline
\end{tabular}

Fuente: Flores S. (2009). Tratamiento de Remediación de Efluentes Metalúrgicos con énfasis en el abatimiento de cobre con dolomita. Tesis para obtener Titulo Profesional de Ingeniero Metalúrgico de la Universidad Nacional Mayor de San Marcos. ${ }^{[6]}$

\subsubsection{Caracterización mineralógica por micros- copía electrónica de barrido}

3.2.2.1. Caracterización mineralógica por microscopía electrónica de barrido de dolomita natural 
Figura 1. Micrografía de microscopía electrónica de barrido de dolomita natural

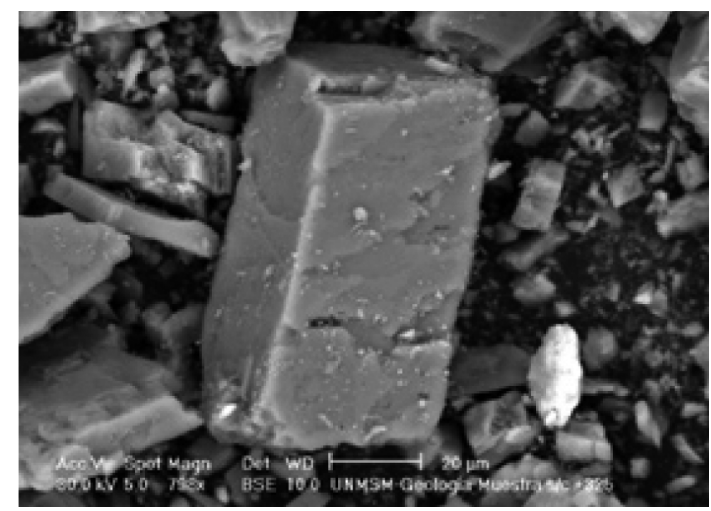

Fuente: Flores S. (2009). Tratamiento de Remediación de Efluentes metalúrgicos con énfasis en el abatimiento de cobre con dolomita. Tesis para obtener Titulo Profesional de Ingeniero Metalúrgico de la Universidad Nacional Mayor de San Marcos. ${ }^{[6]}$

Figura $N^{\circ}$ 2. Respuesta espectral de difracción de rayos $\mathrm{X}$ de caracterización mineralógica por microscopía electrónica de barrido de dolomita natural

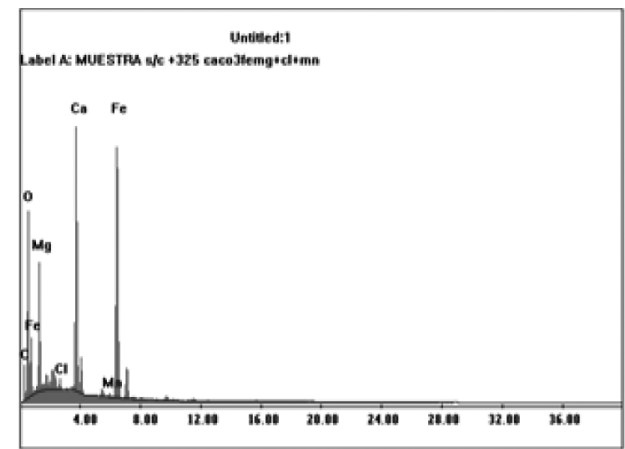

Fuente: Tesis para obtener Titulo Profesional de Ingeniero Metalúrgico de la Universidad Nacional Mayor de San Marcos, 2009. Flores S. "Tratamiento de remediación de efluentes metalúrgicos con énfasis en el abatimiento de cobre con dolomita" ${ }^{[6]}$.

\subsubsection{Caracterización mineralógica de dolomita} comercial de agregados calcáreos por microscopía electrónica de barrido

Figura 3. Micrografía de microscopía electrónica de barrido de dolomita comercial

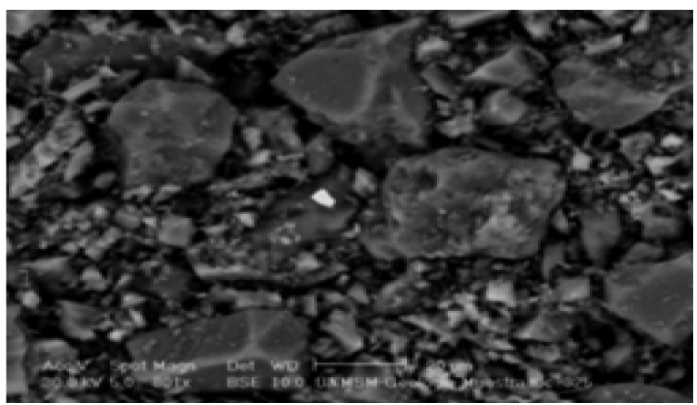

Fuente: Flores S. (2009). Tratamiento de Remediación de Efluentes metalúrgicos con énfasis en el abatimiento de cobre con dolomita. Tesis para obtener Titulo Profesional de Ingeniero Metalúrgico de la Universidad Nacional Mayor de San Marcos ${ }^{[6]}$.
Figura 4. Respuesta espectral de difracción de rayos $\mathrm{X}$ de caracterización mineralógica por microscopía electrónica de barrido de dolomita natural

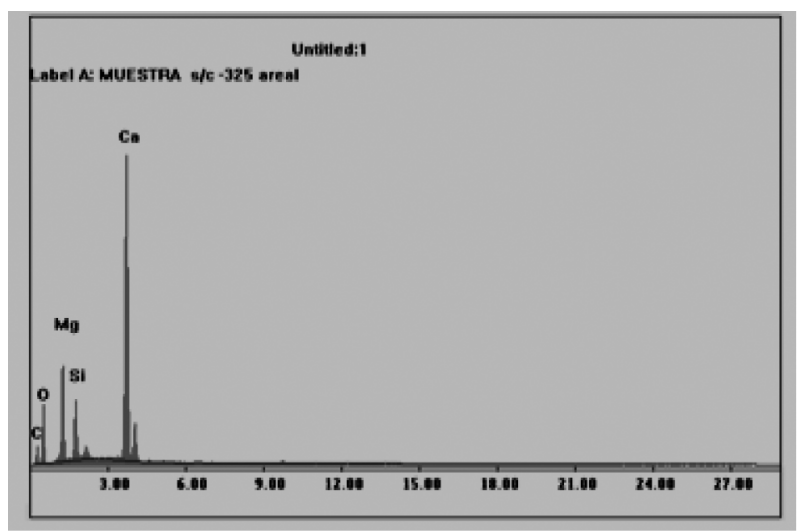

Fuente: Flores S. (2009). Tratamiento de Remediación de Efluentes metalúrgicos con énfasis en el abatimiento de cobre con dolomita. Tesis para obtener Titulo Profesional de Ingeniero Metalúrgico de la Universidad Nacional Mayor de San Marcos ${ }^{[6]}$.

\subsection{Caracterización del lodo de efluente de flotación}

\subsubsection{Naturaleza del efluente metalúrgico}

El efluente metalúrgico empleado en las pruebas experimentales proviene de la planta concentradora de la Universidad Nacional de Ingeniería.

\subsubsection{Análisis químico}

En la primera parte de la investigación que comprende la realización de las pruebas experimentales, solo se realizó análisis químico del cobre, el cual tiene una concentración inicial de $0.34 \mathrm{~g} / \mathrm{lt}$, para efluente de flotación.

\subsubsection{Caracterización de lodos de efluentes me- talúrgicos por difracción de rayos $\mathrm{X}$}

Figura 5. Caracterización de lodos de efluentes metalúrgicos por difracción de rayos $\mathrm{X}$

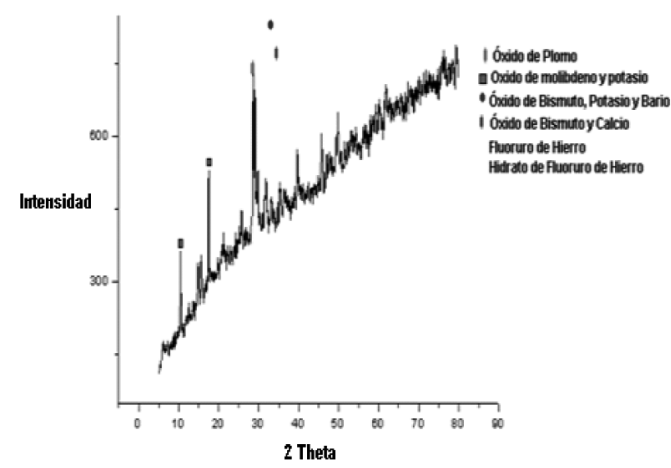

Fuente: Flores S. (2009). Tratamiento de Remediación de Efluentes metalúrgicos con énfasis en el abatimiento de cobre con dolomita. Tesis para obtener Titulo Profesional de Ingeniero Metalúrgico de la Universidad Nacional Mayor de San Marcos ${ }^{[6]}$. 


\section{PRUEBAS EXPERIMENTALES DE REMEDIA- CIÓN DE EFLUENTES DE FLOTACIÓN CON DO- LOMITA}

\subsection{Pruebas experimentales de remediación de efluente de flotación con dolomita natural}

\subsubsection{Efluente metalúrgico de flotación tratado con dolomita natural tratada (ver tabla 3 ).}

Tabla 3. Cinética de adsorción de cobre con dolomita natural tratada

\begin{tabular}{|c|c|}
\hline $\begin{array}{c}\text { Tiempo } \\
\text { (Minutos) }\end{array}$ & $\begin{array}{c}\text { Concentración de co- } \\
\text { bre en sistema (gr/Lt) }\end{array}$ \\
\hline 0 & 0.561 \\
\hline 5 & 0.154 \\
\hline 10 & 0.031 \\
\hline
\end{tabular}

Fuente: Pruebas de Laboratorio de Tesis de Flores S. "Tratamiento de remediación de efluentes metalúrgicos con énfasis en el abatimiento de cobre con dolomita", para obtener Titulo Profesional de Ingeniera Metalúrgica de la UNMSM, 2009. ${ }^{[4]}$

\section{Condiciones de la prueba}

Dolomita: Natural.

$\mathrm{pH}$ inicial de la solución de lixiviación: 3

$\mathrm{pH}$ final de la solución de lixiviación: 14

Porcentaje de reducción de cobre: $94.47 \%$

\subsection{Pruebas experimentales de remediación de efluente de flotación con dolomita comercial}

\subsubsection{Efluente metalúrgico de flotación tratado con dolomita comercial (ver Tabla 4)}

Tabla 4. Cinética de adsorción de cobre con dolomita comercial tratada.

\begin{tabular}{|l|c|}
\hline \multicolumn{1}{|c|}{$\begin{array}{c}\text { Tiempo } \\
\text { (Minutos) }\end{array}$} & $\begin{array}{c}\text { Concentración de cobre } \\
\text { en sistema }(\mathrm{g} / \mathrm{Lt})\end{array}$ \\
\hline 0 & 0,519 \\
\hline 15 & 0,099 \\
\hline 20 & 0,054 \\
\hline 30 & 0,025 \\
\hline
\end{tabular}

Fuente: Pruebas de Laboratorio de Tesis de Flores S. Tratamiento de remediación de efluentes metalúrgicos con énfasis en el abatimiento de cobre con dolomita, para obtener Titulo Profesional de Ingeniero Metalúrgico de la UNMSM, 2009. ${ }^{[4]}$

Condiciones de la prueba

Dolomita: Agregados calcáreos

$\mathrm{pH}$ inicial de la solución de lixiviación: 3

$\mathrm{pH}$ final de la solución de lixiviación: 13

Porcentaje de reducción de cobre: 95.18\%.

\section{DISCUSIÓN DE RESULTADOS}

Las pruebas experimentales de remediación de efluentes metalúrgicos demostraron los siguientes resultados:

\subsection{Sistema: dolomita natural tratada en efluen- te metalúrgico de flotación}

La cinética de adsorción de cobre con dolomita en el sistema muestra que existe reducción de la concentración de cobre. Puesto que la concentración inicial de cobre, cuyo valor es de $0.561 \mathrm{~g} / \mathrm{tt}$, disminuye cuando el sistema es sometido durante 10 minutos de agitación, cuya concentración de cobre tendrá un valor de $0.031 \mathrm{~g} / \mathrm{lt}$. En ese sentido, el valor del porcentaje de reducción de cobre es de $94,47 \%$.

\subsection{Sistema: dolomita comercial tratada en efluente metalúrgico de flotación}

La cinética de adsorción de cobre con dolomita en el sistema muestra que existe reducción de la concentración de cobre. Puesto que la concentración inicial de cobre cuyo valor es de $0,519 \mathrm{~g} / \mathrm{lt}$ disminuye cuando el sistema es sometido durante 30 minutos de agitación, cuya concentración de cobre tendrá un valor de $0.025 \mathrm{~g} / \mathrm{lt}$. El valor del porcentaje de reducción de cobre es de 95,18\%.

\section{CONCLUSIONES}

- La dolomita funciona como adsorbente de cobre y otros iones generadores de acidez en efluentes metalúrgicos de flotación y de lixiviación.

- Las pruebas experimentales del tratamiento de remediación de efluentes metalúrgicos emplearon dos tipos de dolomita: dolomita natural y dolomita comercial, cada una de las cuales posee diferente tamaño de partícula. La dolomita natural posee un tamaño de partícula de 74 micrones, mientras que la dolomita comercial posee un tamaño de partícula de 44 micrones.

- El tamaño de partícula de cada una de las dolomitas es una variable independiente de operación, puesto que interviene de manera directa en el tratamiento de remediación de los efluentes metalúrgicos empleados.

- El "Efluente de la UNI", que fue tratado con dolomita natural tratada, presenta porcentaje de reducción de cobre, con un valor de 94,47\%, puesto que la concentración inicial de cobre en efluente de flotación fue de 0,561 g/lt (561 ppm) y luego de ser tratado con dolomita, su concen- 
tración se redujo hasta el valor de 0,031 g/lt (31 ppm).

- El “EFLUENTE DE LA UNI”, que fue tratado con dolomita comercial tratada, presenta porcentaje de reducción de cobre, con un valor de 95,18\%, puesto que la concentración inicial de cobre en efluente de flotación fue de 0,519 g/lt (561 ppm) y luego de ser tratado con dolomita, su concentración se redujo hasta el valor de 0,025 g/lt (31 ppm).

\section{REFERENCIAS BIBLIOGRÁFICAS}

1. Bixio D., y otros. Wastewater Reuse in Europe, "Proceedings Intl. Conf. Integrated Concepts in Water Recycling", Wollongong, NSW Australia, February 14-17, 2005.

2. Durham B., Kim J.B., y Jeong H. Integrated Water Cycle Management-International reuse case studies that demonstrate the benefits, "Proceedings IWCM\&reuse, IWA Reuse conference," Korea 2005.

3. European Integrated Pollutions Prevention and Control Bureau, Reference Document on Best Available Techniques (BREF) on Common Was- te Water and Waste Gas Treatment/Management Systems in the Chemical Sector, Feb.2003.

4. U.S. Environmental Protection Agency, Guidelines for water reuse, EPA/625/R-04/108, September, 2004.

5. J. Le Pol y otros Storm water reuse: the onsite operating experience of the car factory of Maubeuge Construction Automobile. "Proceedings of the Novatech Conference”. France, 2001.

6. Flores S. Tratamiento de remediación de efluentes metalúrgicos con énfasis en el abatimiento de cobre con dolomita. Tesis para obtener el Título de Ingeniero Metalúrgico de la Universidad Nacional Mayor de San Marcos.

7. Romero A. "Estudio de los metales pesados en el relave minero abandonado de Ticapampa". Paper de Revista de Instituto de Investigación de la Facultad de Ingeniería Geológica, Minera, Metalúrgica, Geográfica de la Universidad Nacional Mayor de San Marcos. Vol. 11 N. ${ }^{\circ} 22$, pp. 13-16.

8. Aduvire, O. y Aduvire, H. 2005. Aguas ácidas de mina: caracterización, mineralogía y microbiología. Revista INGEOPRES 141. Madrid (España). 52-62. 